\title{
Nonrelativistic QCD for colour scalar fields
}

\author{
Andre Hoang and Pedro Ruiz-Femenia* \\ Max-Planck-Institut für Physik (Werner-Heisenberg-Institut), Germany \\ E-mail: ahoang@mppmu.mpg.de, ruizfeme@mppmu.mpg.de
}

\begin{abstract}
We present an effective field theory suitable to describe a non-relativistic particle-antiparticle pair of heavy scalars based on the gauge group SU(3). Its formulation is analogous to that of "velocity NRQCD" (vNRQCD), a non-relativistic effective theory for heavy quark pairs. The matching conditions with scalar QCD and the renormalization group evolution of the effective theory are discussed. The non-relativistic framework proposed here suffices to compute scalar-antiscalar bound state energies at next-to-next-to-leading-logarithmic (NNLL) order and next-to-leadinglogarithmic (NLL) threshold production of squarks in $e^{+} e^{-}$and $\gamma \gamma$ collisions, in particular of the lighter scalar top quark at a future Linear Collider.
\end{abstract}

International Europhysics Conference on High Energy Physics

July 21st - 27th 2005

Lisboa, Portugal

* Speaker. 


\section{Introduction}

Supersymmetry (SUSY) requires the existence of two scalar partners (squarks) for every quark corresponding to its two helicity states. Many models of SUSY predict that, due to large mixing, the lightest squark could correspond to one of the mass eigenstates of the third generation with $m_{\tilde{q}}<500 \mathrm{GeV}$, thus allowing for the production of stop pairs at a future $e^{+} e^{-}$Linear Collider operating below $1 \mathrm{TeV}$. A "threshold scan" of the total cross section line-shape at such facility will yield precise measurements of the stop mass, lifetime and couplings, in close analogy to the program carried out in threshold studies for the top-antitop threshold [1]. It is then clear that an accurate description of the shape of the squark production cross section at energies close to threshold will be mandatory to put on equal footing the theoretical and experimental uncertainties.

Close to threshold squark pairs are produced with small velocities in the center of mass frame, $m v^{2} \equiv \sqrt{s}-2 m, m$ being the heavy squark mass. The low-energy QCD dynamics of squarks is based on standard QCD if we assume that the gluino is not much lighter that the electroweak scale. The multi-gluon exchange of time-like gluons produces singular terms at threshold $\propto\left(\alpha_{s} / v\right)^{n}$ and $\propto\left(\alpha_{s} \ln v\right)^{n}$ at the $n$-loop order in QCD perturbation theory, which forces one to carry out a double expansion in $\alpha_{s}$ and $v$ and count $v \sim \alpha_{s}$ to define the power counting in this regime. The singular terms mentioned above must be thus termed as leading order contributions and need to be summed up to all orders in $\alpha_{s}$. For top-antitop pair production at threshold, vNRQCD ("velocity NRQCD") [2, 3] has been shown to be the proper framework to consistently perform the summation to NNLL order [4], which means to account up to $\mathscr{O}\left(\alpha_{s}^{2}, \alpha_{s} v, v^{2}\right)$ corrections to the leadinglogarithmic (LL) order $\propto \sum_{k}\left(\alpha_{s} / v\right)^{k} \sum_{i}\left(\alpha_{s} \ln v\right)^{i}$. An analogous effective field theory describing the non-relativistic interaction between pairs of colored scalars can provide the needed ingredients for a renormalization group improved computation of squark pair production at threshold. A complete summation at NLL order requires the knowledge of the Wilson coefficients of potentials at LL order and of nonrelativistic production currents at NLL order [2, 3]. The effective Lagrangian for the scalar version of vNRQCD (that will be called simply vNRQCD in the following) and the relevant matching conditions and anomalous dimensions are outlined in this letter. We refer the reader to a recent work [5] for a more detailed analysis and explicit formulae.

\section{Effective theory set-up}

The vNRQCD effective theory is formulated by including only those quark and gluonic degrees of freedom which can become on-shell for energies below $m$. The on-shell degrees of freedom correspond to gluons $\left(A_{q}^{\mu}, A^{\mu}\right)$ and ghosts $\left(c_{q}, c\right)$ with soft $\sim(m v, m v)$ and ultrasoft $\sim\left(m v^{2}, m v^{2}\right)$, energy and momenta, and potential heavy squarks $\left(\psi_{\mathbf{p}}\right)$ and antisquarks $\left(\chi_{\mathbf{p}}\right)$, with energy $\sim m v^{2}$ and soft three momentum $\mathbf{p} \sim m v$. Massless quarks and squarks can also be included with soft and ultrasoft components in vNRQCD. The dependences on soft momenta of the heavy squark and soft gluons appear as labels on the fields and an explicit coordinate dependence refers only to ultrasoft fluctuations [2]. All off-shell effects such as those from hard squarks and gluons, potential gluons, and soft quarks are accounted for by on-shell matching of vNRQCD to full QCD at the hard scale $m$. The basic vNRQCD Lagrangian can be separated into ultrasoft, soft and potential components $\mathscr{L}=\mathscr{L}_{u}+\mathscr{L}_{s}+\mathscr{L}_{p}$. The ultrasoft piece of the effective Lagrangian describes the interactions of 


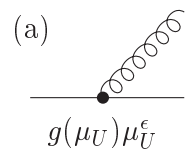

(b)

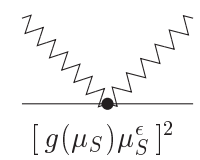

(c)

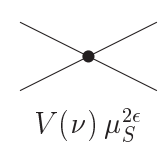

Figure 1: Effective theory diagrams for the interaction of a heavy squark with an ultrasoft gluon.

ultrasoft gluons with squarks (Fig. 1)a, which are multipole expanded to separate ultrasoft momenta from larger momenta, and also contains the kinetic energy contributions for the squarks and the ultrasoft fields:

$$
\mathscr{L}_{u}=\sum_{\mathbf{p}}\left\{\psi_{\mathbf{p}}^{*}\left[i D^{0}-\frac{(\mathbf{p}-i \mathbf{D})^{2}}{2 m}+\frac{\mathbf{p}^{4}}{8 m^{3}}+\ldots\right] \psi_{\mathbf{p}}+(\psi \rightarrow \chi, T \rightarrow \bar{T})\right\}-\frac{1}{4} G_{u}^{\mu v} G_{\mu \nu}^{u}
$$

where $G^{\mu \nu}$ is the ultrasoft field strength tensor and the covariant derivative, $D^{\mu}=\partial^{\mu}+i \mu_{U}^{\varepsilon} g_{u} A^{\mu}$, only involves the ultrasoft gluon field and the ultrasoft gauge coupling $g_{u}=g_{u}\left(\mu_{U}\right)$. The factor of the ultrasoft renormalization scale $\mu_{U}$ that appears in the covariant derivative is determined by the requirement that the kinetic terms in the vNRQCD action are of order $v^{0}$ 边, 的 in $d=4-2 \varepsilon$ dimensions, and we employ the same power counting formalism as in the heavy quark case [2, 3]. Likewise, the velocity scaling determines the factors of the soft renormalization scale $\mu_{S}$ accompanying the soft gauge coupling $g_{s}=g_{s}\left(\mu_{S}\right)$ in the soft and potential interactions (Figs. [1 b,c). The existence of two different subtraction scales guarantees that each of loop in the EFT receives the proper renormalization scale according to the three-momenta flowing through it. A crucial feature of vNRQCD is that $\mu_{S}$ and $\mu_{U}$ are required to be correlated according to the non-relativistic energy-momentum relation of a heavy particle, i.e. $\mu_{U}=\mu_{S}^{2} / m=m v^{2}$, where $v$ is the dimensionless renormalization parameter of vNRQCD. Lowering $v$ from the hard matching scale $(v=1)$ to a scale of order the squark velocity sums all logarithms of the soft and ultrasoft scales into the Wilson coefficients of the vNRQCD operators, and renders vNRQCD matrix elements small.

The vNRQCD Lagrangian also contain operators that describe interactions of quarks with soft gluons $\mathscr{L}_{s}$ (Fig. 1 $1 \mathrm{~b}$ ), that arise when soft quarks are integrated out, and potential-like squarkantisquark interactions $\mathscr{L}_{p}$ (Fig. 1 $1 \mathrm{c}$ ), originating from potential gluons and other off-shell modes. Setting aside spin-dependent contributions that are absent for scalars, the matching coefficients of $\mathscr{L}_{s}$ in the scalar theory agree with the corresponding ones in the quark theory due to the equivalence of the heavy quark and heavy scalar effective Lagrangians (HQET and HSET) at order $1 / \mathrm{m}$. Differences arise at NNLO because the Darwin term vanishes at the hard scale in HSET [5]. The coefficients in $\mathscr{L}_{s}$ are only renormalized due to soft interactions [3, 6] and the structure of the resulting soft divergences is identical to the one in HSET. The potential Lagrangian has terms

$$
\mathscr{L}_{p}=-\mu_{S}^{2 \varepsilon} \sum_{\mathbf{p}, \mathbf{p}^{\prime}} V\left(\mathbf{p}, \mathbf{p}^{\prime}\right) \psi_{\mathbf{p}^{\prime}}^{*} \psi_{\mathbf{p}} \chi_{-\mathbf{p}^{\prime}}^{*} \chi_{-\mathbf{p}}+\ldots \quad, \quad V\left(\mathbf{p}, \mathbf{p}^{\prime}\right)=\left(T^{A} \otimes \bar{T}^{A}\right)\left[\frac{\mathscr{V}_{c}^{(T)}}{\left(\mathbf{p}^{\prime}-\mathbf{p}\right)^{2}}+\ldots\right]
$$

The coefficients $\mathscr{V}^{(1, T)}$ are obtained by matching to the full theory Born scattering diagrams (e.g. for the Coulomb potential shown above, $\left.\mathscr{V}_{c}^{(T)}(1)=4 \pi \alpha_{s}(m)\right)$, and they differ in general from the corresponding results in the quark case. The LL renormalization of the potentials are determined by the effective theory one-loop graphs shown in Fig. Za. Since to the order we are interested in interactions with ultrasoft gluons are not sensitive to spin, the 1-loop ultrasoft renormalization is 
(a)
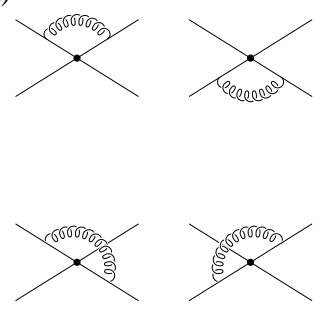
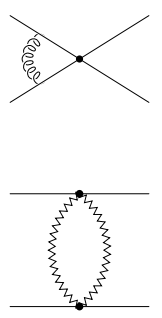

(b)

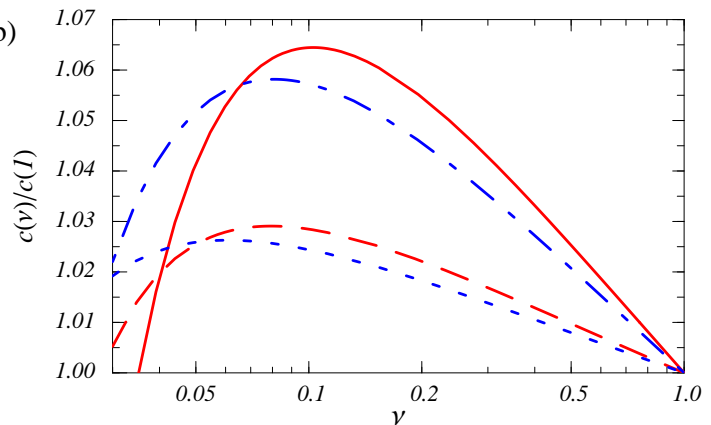

Figure 2: (a) Diagrams for the computation of the LL anomalous dimension of potentials. (b) NLL running of the normalized Wilson coefficient for S-wave and P-wave currents for $m=220 \mathrm{GeV}$ (solid and dashed lines) and for $m=500 \mathrm{GeV}$ (dot-dashed and dotted lines).

the same in vNRQCD for both quarks and squarks. There are a number of operators in the potential and soft parts of vNRQCD with vanishing matching conditions at $v=1$ which however become non-zero for $v<1$ due to ultrasoft renormalization [及].

\section{Anomalous dimensions for production currents}

At leading order in the non-relativistic expansion heavy squark-antisquarks pairs are produced in S- and P-wave states in $\gamma \gamma$ and $e^{+} e^{-}$collisions by the currents $J_{S, \mathbf{p}}=\psi_{\mathbf{p}}^{*} \chi_{-\mathbf{p}}^{*}$ and $\mathbf{J}_{P, \mathbf{p}}=\psi_{\mathbf{p}}^{*} \mathbf{p} \chi_{-\mathbf{p}}^{*}$ respectively, with corresponding Wilson coefficients $c_{s}(v)$ and $c_{P}(v)$. The total cross sections $\sigma\left(e^{+} e^{-}, \gamma \gamma \rightarrow \tilde{q} \tilde{\tilde{q}}\right)$ can be written in terms of the imaginary part of the correlators built from the non-relativistic currents and the products of their Wilson coefficients. The NLL running of the Wilson coefficients is determined by the two-loop anomalous dimensions of the currents, as calculated in Ref. [河]. The results are displayed in Fig.2 $\mathrm{b}$. The scale variation for the $\mathrm{S}$-wave coefficient is found to be much stronger than for the P-wave coefficient and the maxima slightly decrease with the heavy scalar mass and also move towards smaller values of $v$. The remaining scale dependence in the NLL cross section comes from the non-relativistic correlators that are proportional to the zero-distance Green's functions obtained by solving the Schrödinger equation with the NLL improved Coulomb potential.

\section{References}

[1] M. Martinez and R. Miquel, Eur. Phys. J. C 27, 49 (2003); A. H. Hoang et al., in Eur. Phys. J. direct C3, 1 (2000); A. H. Hoang, arXiv:hep-ph/0204299.

[2] M. Luke, A. Manohar and I. Rothstein, Phys. Rev. D 61, 074025 (2000); A.V. Manohar and I.W. Stewart, Phys. Rev. D 62, 074015 (2000).

[3] A. H. Hoang and I. W. Stewart, Phys. Rev. D 67, 114020 (2003).

[4] A. H. Hoang, A. V. Manohar, I. W. Stewart and T. Teubner, Phys. Rev. Lett. 86, 1951 (2001);

A. H. Hoang, A. V. Manohar, I. W. Stewart and T. Teubner, Phys. Rev. D 65, 014014 (2002);

A. H. Hoang, Phys. Rev. D 69, 034009 (2004).

[5] A. H. Hoang and P. Ruiz-Femenia, arXiv:hep-ph/0511102.

[6] A.V. Manohar and I.W. Stewart, Phys. Rev. D 62, 014033 (2000) [arXiv:hep-ph/9912226]. 